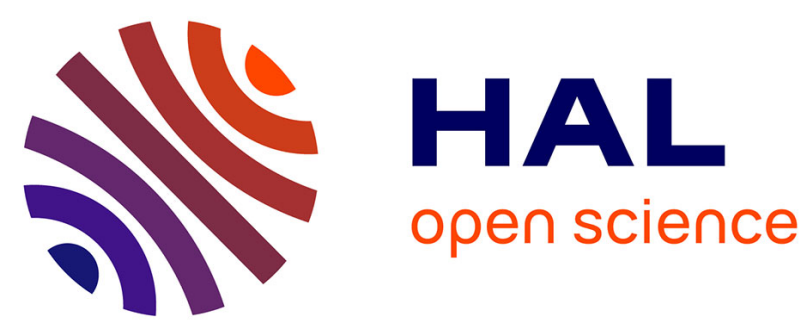

\title{
WETTING ON GELS: HOW THE GEL CHARACTERISTICS AFFECT THE CONTACT LINE DYNAMICS
}

Tadashi Kajiya, Philippe Brunet, Adrian Daerr, Laurent Royon, Tetsuharu

Narita, François Lequeux, Laurent Limat

\section{To cite this version:}

Tadashi Kajiya, Philippe Brunet, Adrian Daerr, Laurent Royon, Tetsuharu Narita, et al.. WETTING ON GELS: HOW THE GEL CHARACTERISTICS AFFECT THE CONTACT LINE DYNAMICS. Interfacial Phenomena and Heat Transfer, 2013, 1 (3), pp.215 - 230. 10.1615/InterfacPhenomHeatTransfer.2013010169 . hal-01550763

\section{HAL Id: hal-01550763 https://hal.science/hal-01550763}

Submitted on 16 Nov 2019

HAL is a multi-disciplinary open access archive for the deposit and dissemination of scientific research documents, whether they are published or not. The documents may come from teaching and research institutions in France or abroad, or from public or private research centers.
L'archive ouverte pluridisciplinaire HAL, est destinée au dépôt et à la diffusion de documents scientifiques de niveau recherche, publiés ou non, émanant des établissements d'enseignement et de recherche français ou étrangers, des laboratoires publics ou privés. 


\title{
Wetting on Gels: How the Gel Characteristics Affect the Contact Line Dynamics?
}

\author{
Tadashi Kajiya ${ }^{(a) \dagger}$, Philippe Brunet ${ }^{(a)}$, Adrian Daerr ${ }^{(a)}$, Laurent \\ Royon $^{(a)}$, Tetsuharu Narita ${ }^{(b)}$, François Lequeux ${ }^{(b)}$ and Laurent $\operatorname{Limat}^{(a)}$ \\ (a) Laboratoire MSC, UMR $705^{r}$ CNRS, \\ Université Paris Diderot, Bâtiment Condorcet, \\ 10 rue Alice Domon et Léonie Duquet, 75205 Paris cedex 13, France \\ (b) PPMD-SIMM, UMR 7615, CNRS, UPMC, ESPCIParisTech, \\ 10 rue Vauquelin, 75231 Paris Cedex 05, France
}

(Dated: November 27, 2013)

\begin{abstract}
In this article, we summarize a series of experimental works and qualitative modelling regarding the dynamics of liquid contact line on gel substrates. Two different situations were investigated, i.e., water on hydrophilic Poly(2-acrylamido-2-methyl-propane-sulfonic acid -co- acrylamide)(PAMPSPAAM) gels and water on hydrophobic Poly(styrene-butadiene-styrene)(SBS)-paraffin gels. In both situations, different gel characteristics largely affect the contact line dynamics: liquid diffusion and surface deformation by capillary force. On hydrophilic gels, the contact line of a sessile droplet exhibits successively two different behaviours: pinned and receding, and the transition between the behaviours is closely related to the local deformation of the gel surface due to swelling. On hydrophobic gels, the contact line exhibits several different regimes of motions, i.e., stick-slip, and two continuous motions. These transitions are characterized by a frequency $f$ built upon the apparent contact line velocity $v$ and the droplet radius $R$ as $f=v / R$, indicating that the gel rheology largely affects the dynamics of liquid contact line. Our results provide a synthetic view of the characteristic features how the wetting is different on gel surfaces. Finally, we designate unsolved problems and future directions.
\end{abstract}

\footnotetext{
$\dagger^{\dagger}$ Current affiliation is Max Planck Institute for Polymer Research, Ackermannweg 10, D-55128 Mainz, Germany
} 


\section{INTRODUCTION}

Gels are intriguing materials that behave either like solids or liquids due to a threedimensional cross-linked networks expanded throughout its whole volume by a fluid [1-3]. Due to their unique properties, they have large number of applications ranging from medical science to pharmaceutical and food chemistry [4-6]. For those applications, the key is the appropriate tuning of liquid/gel interfaces, as they determine adhesion and friction [7-9] (e.g. cartilage replacement), surface tension and wetting properties (e.g. soft contact lens, artificial organ) [10-13].

From the viewpoint of fundamental science, gels are interesting model systems to explore how liquid motion is different on surfaces which are not "ideal" solid. Statics and dynamics of wetting is still an active subject of research even on hard solid surfaces, as we have to consider the local balance of surface forces at the three-phase contact line in addition to classical hydrodynamics $[14,15]$. On gels, the situation would be more complex as the wetting liquid can cause a large deformation on the gel surface which successively affects the statics and dynamics of the contact line. The gel deformation is caused by two mechanisms: by the balance between the interfacial tensions and elastic resistance of the gel and by the volume exchange between the liquid and gel $[16,17]$. Intuitively, the behaviour of the contact line on gels might be understood in analogy with the wetting on soft surfaces like an elastomer [18-21] or with the wetting on permeable surfaces like porous media [22, 23]. However, as gels have an unusual nature between solids and liquids, the wetting on gels would be even more complex than those situations [24].

In this article, we summarize our experimental works and qualitative modelling regarding the contact line dynamics on gels in two different situations: water on hydrophilic gels [25] and on hydrophobic gels $[26,27]$. In both situations, different nature of gels affects the contact line dynamics.

As a first system, the dynamics of water sessile drops placed on hydrophilic Poly (2acrylamido-2-methyl-propane-sulfonic acid -co- acrylamide)(PAMPS-PAAM) gel substrates were studied. The precise analysis of the contact line in the presence of the substrate deformation and liquid diffusion requires to measure both the profiles of the droplet and of gel substrate simultaneously [28, 29]. To obtain these two profiles, we used "grid projection method" [30, 31], i.e., we projected a grid pattern below the gel surface and measured its 
optical distortion to reconstruct the original profile. We observed that as the water droplet diffuses into the gel substrate, the behaviour of the contact line exhibits successively two different regimes: pinned and receding, and the transition between these two regimes is closely related to the local swelling of the gel around the contact line. We discuss how this pinned-receding transition depends on the gel properties, i.e., the rigidity and hydrophilicity (tuned by the concentrations of crosslinking agent and of hydrophilic AMPS monomer).

The other aspect of the gel complexity is the rheology. As a second system, we studied the dynamics of the moving contact line on poly(styrene-butadiene-styrene)(SBS)-paraffin gel substrates in two geometries: advancing drops and dip-coating. As the SBS-paraffin gel is hydrophobic and there is no volume exchange between the drop and gel, the sole possible effect affecting the contact line is the gel surface deformation due to the capillary force of liquid [18, 19] moderated by the viscoelastic response of the material. However, unlike the case of wetting on elastomer, the contact line exhibits quite complex behaviours, i.e., it shows two regimes of continuous advancing motion and one of stick-slip motion. The stick-slip motion of the contact line was previously observed by $\mathrm{Pu}$ et al. during the wetting on thin polymer films [32, 33]. Here, we found that on a SBS-paraffin gel, there are three different regimes, i.e., continuous, stick-slip, and another continuous motion. We discuss how the transitions of these contact line motions are characterized by the parameters such as the frequency of the contact line motion, and propose a qualitative modelling.

\section{WATER DROPS ON HYDROPHILIC GELS: EFFECT OF LIQUID DIFFU- SION [25]}

\section{A. Experiment}

Poly (2-acrylamido-2-methyl-propane-sulfonic acid -co- acrylamide) (PAMPS-PAAM) gels were used for substrate, and distilled water (Milli-Q Integral; Millipore, USA) was used for liquid drops. The PAMPS-PAAM gels were prepared through the radical polymerization of a solution of 2-acrylamido-2-methyl-propane-sulfonic acid (AMPS; Sigma-Aldrich, USA) and acrylamide (AAM; Alfa Aesar, USA) in water with a cross-linking agent, $N, N^{\prime}$ methylenebisacrylamide (MBA; Sigma-Aldrich, USA), and initiators, Potassium persulfate (PS; Sigma-Aldrich, USA) and $N, N, N^{\prime}, N^{\prime}$-Tetramethylenediamine (TEMED; Sigma- 
Aldrich, USA). The total molar concentration of monomer was fixed at $1 \mathrm{M}$. The concentrations of AMPS and of crosslinking agent MBA with respect to the total amount of monomer were tuned as control parameters. The dimension of gel samples were $70 \mathrm{~mm}$ in length, 20 $\mathrm{mm}$ in width and $4.5 \mathrm{~mm}$ in thickness.

Figure 1 (a) shows the setup for the drop-gel profiles measurement. The gel substrate was placed on a hollow stage, and a droplet of volume $1 \mu$ l was placed on the substrate with a micropipet. To measure both the profiles of the droplet and of gel simultaneously, a grid projection technique was used [30,31]. In this technique, the profiles are obtained by tracing the distortion of grid lines between before and after the placement of the droplet. The original grid plate was located far from the observation system. The illumination light emitted from the photodiode passed through the grid plate and was converted to a parallel light by an optical lens $(f=200 \mathrm{~mm})$. Then the light was guided to the bottom of the substrate, and passed through a focus lens (TV lens $f=35 \mathrm{~mm}$ : Pentax, Japan). This focus lens projects the image of the grid inside the gel substrate, which is set just below the droplet. The grid image was measured by a CCD camera (A101FC; Basler AG, Germany) which was located above the droplet. The example of the grid image obtained after the placement of droplet is also shown in fig. 1 (b).

The original profile was reconstructed by tracing the light path which passes through each grid line. As shown in fig. 1 (c), the shift of the grid line $d_{s}$ is related to the local slope of the interface between the media of different refraction ratios (medium A: air, medium B: water or gel). The relation between the shift and slope is given by the following three equations:

$$
\begin{gathered}
\sin \alpha=n \sin \beta, \\
\delta=\alpha-\beta, \\
\tan \delta=\frac{d_{s}}{e},
\end{gathered}
$$

where $\alpha$ and $\beta$ are the angles of the light path in media $\mathrm{A}$ and $\mathrm{B}$ with respect to the normal to the interface, $\delta$ is the angle of the light path in medium $\mathrm{B}$ with respect to the vertical axis, $n$ is the refractive index of medium B (since the water volume fraction in the gel is considerably large, we used the value of water $n=1.33$ ). By solving eqs. (1) - (3), 
the local slope of the interface was obtained from $d_{s}$, and the whole profile was obtained by integrating in a horizontal direction $x$.

\section{B. Results}

Figure 2 (a) shows the half cross sections of the profiles (the height $h$ against the radial position $r$ ) of the droplet and gel substrate at different times (Substrate: $C_{M B A}=5 \mathrm{~mol} \%$ and $C_{A M P S}=30 \mathrm{~mol} \%$ ). During the diffusion process of the droplet into the gel substrate, both the profiles of the droplet and substrate change. At an early stage $(t=25 \mathrm{~s})$, the contact line of the droplet is seen clearly, i.e., the slope of the profile is discontinuous at the droplet perimeter. As the water diffusion proceeds, the height of the droplet decreases, while the height of the gel substrate around the contact line increases. The horizontal extent of the substrate deformation grows close to the order of $1 \mathrm{~mm}$ from the initial position of the contact line, and the boundary between the droplet and substrate becomes less clear.

At a late stage, we detected the position of the contact line using the local curvature of the profile at the center $(r \approx 0)$. When a water droplet still remains on the gel surface, the surface of the center where the droplet resides must be a spherical cap of uniform curvature. Therefore, if the local curvature at the center $H_{c}$ is calculated as:

$$
\left.\frac{1}{H_{c}} \approx \frac{\partial^{2} h}{\partial r^{2}}\right|_{r \approx 0}
$$

and is extrapolated outward, the contact line can be detected as the point where the actual profile deviates from the extrapolated $H_{c}$ curve. The extrapolated $H_{c}$ curves are also plotted in fig. 2 (a) as dashed lines.

Now that the position of the contact line has been detected, it is possible to measure the radius of the droplet $R$, the effective contact angle of the droplet $\theta_{\text {drop }}$ with respect to horizontal, and the angle of the local slope of the gel surface $\theta_{\text {gel }}$ close to the contact line. (The determination of $R, \theta_{\text {drop }}$ and $\theta_{\text {gel }}$ are illustrated in the inset of fig. 2 (a).) Figure 2 (b) shows the plot of $R$ against the time $t$, and fig. 2 (c) shows the plot of $\theta_{\text {drop }}$ and $\theta_{\text {gel }}$ against $t$. In both figures, data of substrates: $C_{A M P S}=30 \mathrm{~mol} \%$ and $C_{M B A}=5 \mathrm{~mol} \%$ are shown.

The behaviour of the contact line exhibits two different regimes. The contact line is initially pinned, then at a time $t=t_{r e c}$, it starts receding until the droplet has totally diffused into the gel. By comparing fig. 2 (b) with (c), it is clearly observed that the 
transition of the pinning to receding regimes is closely related to the variation of the angles $\theta_{d r o p}$ and $\theta_{\text {gel }}$. At the initial stage where the contact line is pinned, the values of $\theta_{\text {drop }}$ and $\theta_{\text {gel }}$ are largely different. As the water diffusion proceeds, these two angles come close to each other: $\theta_{\text {drop }}$ decreases while $\theta_{\text {gel }}$ increases. At the moment where $\theta_{\text {drop }}$ and $\theta_{\text {gel }}$ almost correspond, the contact line starts to recede. This result indicates that when a contact line recedes on a PAMPS-PAAM hydrogel, apparently it has a finite receding contact angle, but the actual receding angle on the deformed gel surface $\theta_{d r o p}-\theta_{\text {gel }}$ is nearly $0^{\circ}$. The same trend was observed for all AMPS and MBA concentrations in our experiment.

We also explored how the pinned-receding transition of the contact line depends on the following parameters: the MBA concentration $C_{M B A}$ that determines the rigidity of the gel substrate and the PAMPS concentration $C_{A M P S}$ that determines the hydrophilicity of the substrate. In fig. 3 (a), we plot the relative ratio of initial pinning time to the total diffusion time $t_{r e c} / t_{f}$ on gels of various $C_{M B A}$ and $C_{A M P S}$. For both $C_{A M P S}, t_{r e c} / t_{f}$ becomes longer with the increase of $C_{M B A}$, i.e., the contact line is pinned for a longer time as the gel becomes more rigid. Comparing the data of different $C_{A M P S}, t_{r e c} / t_{f}$ is shorter for higher $C_{A M P S}$ (30 mol\%) in a whole range of $C_{M B A}$, indicating that the contact line recedes earlier when the gel substrate has higher hydrophilicity.

Indeed, both the parameters $C_{M B A}$ and $C_{A M P S}$ largely affect the swelling ability of the gel, i.e., the volume swelling ratio $V_{g e l}^{s w} / V_{\text {gel }}^{i}$ where $V_{g e l}^{s w}$ corresponds the volume of the gel at the fully swollen state and $V_{g e l}^{i}$ the volume at the initial state. Considering that, the data $t_{r e c} / t_{f}$ for all gels are replotted as a function of $V_{g e l}^{s w} / V_{g e l}^{i}$ in fig. $3(\mathrm{~b})$. It is clearly observed that $t_{r e c} / t_{f}$ has an universal negative dependence upon $V_{g e l}^{s w} / V_{g e l}^{i}$.

\section{Discussion}

In this section, we discuss why the actual contact angle of the droplet $\Delta \theta=\theta_{\text {drop }}-\theta_{\text {gel }}$ becomes nearly $0^{\circ}$ at the moment of the contact line recession. Figure 4 shows the schematics of the mechanism we propose.

After the droplet is placed on a gel substrate, the water starts to diffuse from the droplet into the substrate, and the gel deforms as it swells with water. Here, we consider the diffusion of water into the gel with an analogy of the drying problems of the droplet [34-36]. Due to the geometrical effect, it is expected that the diffusive flux of water near the edge of the 
droplet is largely enhanced compared to the center (fig. 4 (a)). Therefore, the gel surface below the contact line swells with water rapidly, forming a "locally swollen" region. For the contact line to recede, the actual contact angle of the droplet $\Delta \theta=\theta_{\text {drop }}-\theta_{\text {gel }}$ must correspond to the equilibrium contact angle on the swollen gel surface.

To check the wetting property of the swollen gel surface, we conducted a supplemental wetting experiment on a gel substrate which is previously fully swollen in a water bath. We observed that on a fully swollen gel, the droplet spreads rapidly and the contact angle takes a considerably small value $\left(<3^{\circ}\right)$ irrespective of $C_{M B A}$ and $C_{A M P S}$. The results of the supplemental experiment implies that the equilibrium contact angle on the locally swollen gel surface is very close to $0^{\circ}$. This is consistent with the result that the contact line of the droplet is pinned until $\Delta \theta$ reaches nearly $0^{\circ}$.

The negative dependence of $t_{r e c} / t_{f}$ on the volume swelling ratio of the substrate $V_{g e l}^{s w} / V_{g e l}^{i}$ in fig. 3 (c) can be understood in terms of the growth of the deformation of the gel surface. For a gel substrate with a large swelling ratio, the gel is highly permeable to water. After a droplet is placed on the substrate, water permeates into the gel rapidly and the region near the water-gel interface are swollen by the flux, which causes the large rise of the gel surface around the droplet. Therefore, the local slope of the gel surface at the contact line $\theta_{\text {gel }}$ increases up to the value of $\theta_{\text {drop }}$ at an early stage of the diffusion process. On the other hand, for a gel with a small swelling ratio, the gel exhibits low permeability and thus the surface deformation by swelling is tiny. In that case, $\theta_{\text {drop }}$ does not corresponds to $\theta_{\text {gel }}$ until the droplet diffuses most of water into the gel and decreases $\theta_{\text {drop }}$ to a small value.

To get the order of magnitude for the $\theta_{\text {drop }}$ and $\theta_{\text {gel }}$ at the initial pinned-contact line stage,we propose a simple model depicted in fig. 4 (b). We assume that below the droplet, the water diffusion creates the diffusive boundary layer of typical thickness $\sqrt{D t}$, where $D$ is the diffusion coefficient of water in the gel. The typical volume flux of water at the water-gel interface $z=z_{\text {gel }}$ is scaled as

$$
J \sim D \frac{\phi_{s w}-\phi_{i}}{\sqrt{D t}}
$$

where $\phi_{s w}$ is the volume fraction of water in the fully swollen gel (suppose that at the watergel interface, gel is immediately swollen), and $\phi_{i}$ is the volume fraction of water in the gel at the initial state. Due to this flux, the volume of the droplet $V_{d r o p} \approx \pi R^{3} \theta_{\text {drop }}$ decreases as

$$
\frac{d V_{d r o p}}{d t} \approx \pi R^{3} \frac{d \theta_{d r o p}}{d t}=-\pi R^{2} J
$$


while the volume of the gel increases as

$$
\frac{d V_{g e l}}{d t} \approx \pi R^{2} \frac{d z_{g e l}}{d t}=\pi R^{2} J
$$

The local slope of the gel near the contact line $\theta_{\text {gel }}$ is estimated to be close to the ratio $z_{\text {gel }} / d_{\text {gel }}$, where $z_{\text {gel }}$ designates the vertical displacement of the droplet basis due to swelling, and $d_{g e l}$, the horizontal distance on which this displacement relaxes radially around the droplet. With an analogy of a contact problem of solid bodies [37], here we assume that the shape of the gel surface is mainly dictated by a compromise between the shear elasticity of the gel and the rising condition $z=z_{\text {gel }}$ at the droplet basis of radius $R$. The characteristic size of $d_{g e l}$ is thus estimated as the same order of the droplet radius: $d_{\text {gel }} \sim R$.

Combining eqs. (5) - (7), the time evolution of the angles $\theta_{d r o p}$ and $\theta_{\text {gel }}$ are obtained as

$$
\begin{gathered}
\theta_{\text {drop }}(t)-\theta_{\text {drop }}^{(0)} \sim-\frac{\phi_{s w}-\phi_{i}}{R} \sqrt{D t} . \\
\theta_{\text {gel }}(t) \sim \frac{\phi_{s w}-\phi_{i}}{R} \sqrt{D t} .
\end{gathered}
$$

where $\theta_{d r o p}^{(0)}$ is the initial value of the effective contact angle of the droplet. Equations (8) and (9) predict that the time evolutions of $\theta_{\text {drop }}(t)-\theta_{d r o p}^{(0)}$ and of $\theta_{\text {gel }}(t)$ are proportional to $\sqrt{t}$. From eqs. (8) and (9), the characteristic time of the contact line recession $\tau$, i.e., the time when the actual contact angle $\Delta \theta=\theta_{\text {drop }}-\theta_{\text {gel }}$ goes to zero, is thus estimated as

$$
\tau \sim \frac{\left(R \theta_{d r o p}^{(0)}\right)^{2}}{D\left(\phi_{s w}-\phi_{i}\right)^{2}} .
$$

Let us calculate $\tau$ for our gel $\left(C_{M B A}=5 \mathrm{~mol} \%, C_{A M P S}=10 \mathrm{~mol} \%\right): R=1.42 \mathrm{~mm}$, $\theta_{\text {drop }}^{(0)}=27.1^{\circ}, \phi_{s w}-\phi_{i}=0.0529$ (obtained by the measurement of swelling ratio) and $D=39.47 \times 10^{-8} \mathrm{~m}^{2} / \mathrm{s}$ (cited from ref. [38]). By substituting these values in eq. (10), $\tau$ is calculated as ca. 400 s. Experimentally, the time for the onset of the contact line recession was measured as $140 \mathrm{~s}$, which is not far from our theoretical estimation.

\section{MOVING CONTACT LINE ON HYDROPHOBIC GELS: EFFECT OF VISCO- ELASTO-CAPILLARY DEFORMATION $[26,27]$}

\section{A. Experiment}

Poly(styrene-butadiene-styrene)(SBS)-paraffin gels were used for the gel substrates and distilled water (Milli-Q Integral; Millipore, USA) was used for the liquid. SBS powders 
(G1682; Kraton Polymers, USA) were dissolved in paraffin (Norpar15; ExxonMobil, USA) heated in a water bath at $90^{\circ} \mathrm{C}$. After SBS powders were completely dissolved, the solution was poured into a gel mold and was cooled down to ambient temperature. The mass concentration of SBS $c_{S B S}$ was varied from $8 \%$ to $25 \%$. The gel mold consists of two glass plates separated by a rubber spacer. The dimension of gel samples were $70 \mathrm{~mm}$ in length, $20 \mathrm{~mm}$ in width and $2 \mathrm{~mm}$ in thickness. The rheology of the gel was measured by a strain controlled rheometer (Physica MCR 500; Anton Paar, Austria). Shear strain amplitude was set to $1 \%$, and the experiments were conducted at frequencies from $10^{-4} \mathrm{~Hz}$ to $10 \mathrm{~Hz}$.

Figure 5 (a) shows a schematic of the inflation experiment of a water droplet on the gel. A water droplet was placed on a gel by a micro-syringe. The syringe was connected to a motor syringe pump (Model 33; Harvard Apparatus, USA), and it supplies water to the droplet and inflate it at a constant volume rate q. From the side and top of the droplet, the shape of the droplet was monitored by 2 CCD cameras (Model A101fc and Model PLA1000; Basler, Germany) with magnification lenses (CCTV lens; Pentax, Japan).

We investigated the contact line dynamics in another geometry, a dip-coating experiment (see fig. 5 (b)) that allows an accurate control of the mean receding velocity and to operate with a two-dimensional contact line. In the dip-coating, the gel substrate was mounted on a translation stage and withdrawn from a liquid bath at a constant translation velocity $v$. The front view of the contact line and the side view of the meniscus was monitored by two CCD cameras. To enable the visualization of the meniscus, the stage was inclined at $15^{\circ}$ against the vertical axis.

\section{B. Results}

Figure 6 (a) shows sequential pictures and (b) shows the plot of radius and contact angle of a water droplet being inflated on a SBS-paraffin gel of $c_{p o l}=10 \%$ at an inflation rate $q$ $=20 \mu \mathrm{l} / \mathrm{min}$. During the inflation process, the droplet contact line exhibits continuous and stick-slip motions. At an early stage of the inflation process $(t<160 \mathrm{~s})$ while the droplet radius $R$ is still sufficiently small $(R<2 \mathrm{~mm})$, the contact line advances continuously at a nearly constant contact angle of order $100^{\circ}$. As $R$ becomes large at a later stage $(t>160$ $\mathrm{s}, R>2 \mathrm{~mm})$, the contact line starts the stick-slip motion, i.e., the droplet radius $R$ stays at the same value during a certain moment and then suddenly increases. Looking at the 
contact angle, once the contact line sticks, $\theta$ starts to increase. At the moment $\theta$ reaches a critical value of order $100^{\circ}$, the contact line slips forward. Successively, the contact line repeats this stick-slip motion. After the inflation process, the water droplet was removed quickly and a picture of the gel surface was taken as shown in fig. 6 (c). The contact line leaves multiple circular traces on the gel surface.

In the dip-coating experiment, the contact line also exhibits a stick-slip behaviour. Pictures of the contact line motion is shown in fig. 7 (a), and the plots of contact line position and contact angle are shown in fig. 7 (b). Gel of $c_{p o l}=10 \%$ is used and the translation velocity $v$ is fixed at $0.01 \mathrm{~mm} / \mathrm{s}$. In the dip-coating, the stick-slip motion is more periodic. While the substrate is withdrawn from the liquid bath, the contact line is pinned until $\theta$ reaches a critical value close to $40^{\circ}$, then the contact line slips down over a distance approximately $1 \mathrm{~mm}$ and $\theta$ goes back to a value of order $70^{\circ}$. After the slip, it is observed that the contact line leaves a straight line trace on the gel surface.

For a quantitative analysis, we conducted the inflating experiments of water droplets for various volume rates $q$ ranging from from $1 \mu \mathrm{l} / \mathrm{min}$ to $200 \mu \mathrm{l} / \mathrm{min}$. Figure 8 (a) summarizes the contact line behaviour at various $R$ and $q$.

- (i) At high $q$ (e.g., $200 \mu \mathrm{l} / \mathrm{min}$ ), the contact line advances continuously with a constant contact angle during the whole inflation process.

- (ii) At intermediate $q$ (e.g., $20 \mu \mathrm{l} / \mathrm{min}$ ), the contact line initially exhibits the continuous advancing motion. As the droplet radius reaches a critical value $(R \approx 2.2 \mathrm{~mm})$, the contact line starts the stick-slip motion.

- (iii) At low q (e.g., $2 \mu \mathrm{l} / \mathrm{min}$ ), the contact line exhibits the stick-slip motion even at a very early stage of the inflation. However, when the droplet radius increases, now the contact line stops the stick-slip motion and starts to advance continuously again.

The same kinds of transitions of the contact line motions (continuous - stick-slip - continuous) were observed in dip-coating, by varying the translation velocity from $2 \mathrm{~mm} / \mathrm{s}$ to $0.1 \mu \mathrm{m} / \mathrm{s}$.

To explore the local deformation of the gel surface at the contact line, we conducted a supplemental experiment. A sessile droplet of volume $7.5 \mu \mathrm{l}$ was placed on a SBS-paraffin gel and the surface profile near the contact line was measured by an optical profiler (Microsurf $3 \mathrm{D}$, Fogal Nanotech, France). Figure 9 shows the surface profiles of gels $\left(c_{S B S}=10 \%\right)$ at times $t=2,6,10 \mathrm{~min}$. 
The gel surface is pulled up and a ridge is formed at the contact line. Since there is no volume exchange between the droplet and gel, the surface ridge is considered due to the balance between the vertical component of liquid surface tension and the elastic resistance of the gel in analogy with the wetting problem on elastomer. In refs. [39, 40], Shanahan et al. predicted that the elastic surface ridge due to the capillary force has a logarithmic profile that typically leads

$$
\xi(x) \approx \frac{\gamma \sin \theta}{2 \pi G} \ln \left(\frac{d}{|x|}\right),
$$

where $G$ is a shear modulus of the gel, $\theta$ is a droplet contact angle, and $\gamma$ is the surface tension of water $\approx 72 \mathrm{mN} / \mathrm{m}$, and $d$ indicates the macroscopic cutoff length of the surface ridge.

In fig. 9, the profile fits well with the logarithmic curves at $2 \mathrm{~min}$ after the deposition. Meanwhile, the profile of the surface ridge is not persistent. If the contact line stays at the same position on the SBS-paraffin gel, the foot of the surface ridge gradually expands outward, and the profile of the ridge becomes less steep.

\section{Discussion}

In order to compare the contact line behaviour in fig. 8 with the gel rheology, we further estimate the characteristic frequency of the contact line motion defined as:

$$
f=\frac{v_{a}}{R} \approx \frac{q}{2 \pi R^{3}}
$$

where $v_{a}$ is the apparent mean contact line velocity at given $R$ and $q . f$ is the inverse of the typical period at which the contact line moves over the size of the surface deformation $R$ at a velocity $v_{a}$ (here we used $R$ as the size of the surface deformation, as in fig. 9 , the width of the surface ridge is of the same order of drop radius).

In fig. 10 (a), we replot the diagram as a function of $f$. It is clearly observed that the three regimes of the contact line motions (i)-(iii) are defined by $f$ with two critical frequencies which characterize the transition: $f_{c 1}$ separates the (i) continuous advancing motion at a high frequency and (ii) stick-slip regime, and $f_{c 2}$ separates the (ii) stick-slip and (iii) continuous advancing at a low frequency. From the comparison with the measurement of gel rheology, it is also observed that the crossover frequency $f_{\text {cross }}$ where the storage and loss modulus correspond with each other $G^{\prime \prime} / G^{\prime}=1$ is between these two critical frequencies. 
Figure 10 (b) shows the plot of two critical frequencies $f_{c 1}$ and $f_{c 2}$ on gels for various $c_{p o l}$ ranging from $8 \%$ to $20 \%$. With the change of $f_{\text {cross }}$, the values of two critical frequencies vary dramatically.

We have conjectured that the observed contact line motions (continuous-stick-slipcontinuous) are the consequence of the mechanical response of the gel to the capillary force applied at the contact line [41]. The transition of the contact line motions (i)-(iii) can be understood in terms of the characteristic frequency $f$, as schematized in fig. 11.

- Regime (i): When the contact line advances quickly and the characteristic frequency is high $\left(f \gg f_{\text {cross }}\right)$, the gel substrate responds as an elastic solid to the motion of the contact line. In this situation, the surface deformation at the contact line is an elastic ridge pulled by the surface tension of liquid, which propagates with the motion of the contact line, resulting in the continuous advancing motion

- Regime (ii): As the contact line velocity decreases and $f$ comes close to the crossover frequency $\left(f \approx f_{\text {cross }}\right)$, now the response of the gel surface is not purely elastic. The elastic resistance of the gel already starts to relax and a part of the surface deformation becomes irreversible. The surface ridge at the contact line behaves something like a "surface defect" which pins the contact line, resulting in the stick-slip motion.

- Regime (iii): In the other extreme situation, when the contact line advances slowly and $f$ is sufficiently low $\left(f \ll f_{\text {cross }}\right)$, the deformation of the gel surface totally relaxes until the contact line moves over a distance of order $R$. In this case, the gel substrate behaves nearly like a sheet of very viscous liquid with respect to the contact line motion [42]. With the movement of the contact line, a capillary flow is driven below the gel surface. The surface deformation is transported forward by this capillary flow, following the motion of the contact line. Therefore, the contact line exhibits a continuous advancing motion again.

In the stick-slip regime (ii), we propose a qualitative modelling that allows to predict the link between jumps of the radius and jumps of the apparent contact angle, inspired by the model of wetting hysteresis on elastomer by Extrand and Kumagai [43]. The schematic of our model is depicted in fig. 12 (a). The variables $R_{b}, \theta_{b}$ and $R_{a}, \theta_{a}$ are the droplet radii and apparent contact angles before and after the contact line slips on the surface ridge, and $\phi$ 
indicates the local slope of the ridge. Considering that the droplet volume $V$ is conserved, the relations of droplet radii and contact angles are expressed as

$$
V=\frac{\pi R_{b}^{3}}{3}\left(2-3 \cos \theta_{b}\right)=\frac{\pi R_{a}^{3}}{3}\left(2-3 \cos \theta_{a}\right) .
$$

We suppose that $\theta_{a}$ is an advancing contact angle on an undeformed surface, and that the difference between $\theta_{b}$ and $\theta_{a}$ is essentially due to the local surface slope. To advance on a inclined surface, the contact line has to take an advancing angle equal to

$$
\theta_{b}=\phi+\theta_{a}
$$

As $\theta_{a}$ is close to $90^{\circ}$ in our situation, the equations (13) and (14) are re-written as a function of the ratio of two radii

$$
\begin{aligned}
\left(\frac{R_{a}}{R_{b}}\right)^{3} & =\frac{2-3 \cos \left(\theta_{a}+\phi\right)}{2-3 \cos \theta_{a}} \\
& \approx 1+\frac{3}{2}\left[\cos \theta_{a}-\cos \left(\theta_{a}+\phi\right)\right] .
\end{aligned}
$$

With an approximation that $\phi$ is sufficiently small, the ratio $R_{a} / R_{b}$ relates to $\phi$ as shown in the following expression

$$
\frac{R_{a}}{R_{b}} \approx 1+\frac{\phi}{2}
$$

This equation links the ratio of successive droplet radii to the local surface slope $\phi$

$$
\phi=\frac{2\left(R_{a}-R_{b}\right)}{R_{b}}=2 \frac{\Delta R}{R_{b}} .
$$

In order to check this model, we used the experimental data of $c_{S B S}=10 \%$ in the stickslip regime (ii) and calculated the quantity $2 \Delta R / R_{b}$ for various stick-slip motions. In fig. 12 (b), we compared it to the value of $\phi=\theta_{b}-\theta_{a}$ extracted from the direct measurement of the contact angle $\theta$. Both quantities are noted nearly equal, which indicates that our model has captured successfully the essential features.

Let us close this section by discussing the fact that $\phi$ remains locked at the value $10^{\circ}$ independently on $f$. This results are bit surprising because in available modeling in eq. (11) $[39,40]$, the local slope of the surface ridge $h^{\prime}(x)$ depends on the elastic modulus $G$ and thus, here, indirectly on $f$, and even diverges near $x=0$. All seems here to happen as if $\phi$ would be selected by some truncation of this profile at a small scale eliminating the contribution of $G$. In recent studies of wetting on elastomer, this truncation was proposed to be ruled 
by the surface tension of the substrate [44-46]. In particular, one of us (L. Limat) modified the Shanahan's approach by means of the substrate surface tension $\gamma_{s}$ [27]. In its simplest form, for a contact angle of $90^{\circ}$, the expression of $\xi(x)$ is given as

$$
\xi(x) \approx \frac{\gamma \sin \theta}{2 \pi G} \ln \left(\frac{d+l_{e}}{|x|+l_{e}}\right),
$$

where $l_{e}=\gamma_{s} / G$ is the elasto-capillary length. The present approach indicates that, when the substrate surface tension is taken into account, this logarithmic is still valid but shift of a distance equal to $l_{e}$ which "cuts" the divergence at this scale, connecting to the Neumann equilibrium at $x=0$ (fig. 12 (c))

$$
2 \gamma_{s} \theta_{s}=\gamma \sin \theta
$$

where $\theta_{s}$ is the local slope of the surface ridge.

For a paraffin gel, the typical value of $\gamma_{s}$ is $30 \mathrm{mN} / \mathrm{m}[47,48]$, giving the value of $l_{e} \approx 6$ $\mu \mathrm{m}$ for $G=5 \mathrm{kPa}$. Indeed, we have checked experimentaly by profilometry (fig. 9) that a slope of order $10^{\circ}$ of the substrate is reached at approximately $10 \mu \mathrm{m}$ from the contact line, which is quite close to the expected value for $l_{e}$. In addition, the profile of the surface in the vicinity of the contact line $(10 \mu \mathrm{m} \leq x \leq 50 \mu \mathrm{m})$ does not evolve significantly within our time window. From these remarks, it is quite natural that $\phi$ does not depend on $f$.

Note however that this model has also a prediction for $\phi$, whose simplest form is $\phi=\theta_{s}$ when the two substrate surface tensions (dry and wetted) are equal. In our case, there is a problem with this approximation, as with the value obtained from the literature for $\gamma_{s}$ $\left(\gamma_{s}=30 \mathrm{mN} / \mathrm{m}\right)$, one has $\gamma>2 \gamma_{s}$. This means that Neumann equilibrium also can not be satisfied at the contact line. Furthermore, we should consider the balance of forces in asymmetric situations, as in our experiment, the advancing and receding angles are different from $90^{\circ}$.

Although the models for the wetting on elastomer can not be applied directly here as the paraffin gel is visco-elastic and the three interfacial tensions cannot be balanced in our situation, our results suggest that one of their main prediction, i.e. the relevance of elastocapillary length at small scale, could also hold in this very complex system. 


\section{CONCLUSION}

In this paper, we report the dynamics of liquid contact line on gel substrates: hydrophilic PAMPS-PAAM gel and hydrophobic SBS-paraffin gels. In both situations, different gel characteristics dramatically affect the contact line behaviour.

On hydrophilic PAMPS-PAAM gels, the contact line of the sessile droplet successively exhibits two different regimes: pinned and receding. The contact line is initially pinned after the droplet is placed. As the water diffusion proceeds, the effective contact angle of the droplet decreases while the local slope of the gel surface near the contact line increases. Finally these two angles almost correspond to each other, and it is at this moment that the contact line starts to recede. Therefore, at the moment of the contact line recession, the actual contact angle is nearly $0^{\circ}$. About the mechanism of the pinning-receding transitions, we have proposed a physical model that the diffusive flux of the liquid forms a locally swollen region at the contact line, and that whether the contact line is pinned or recede is determined by the wetting property of this swollen region. This model correctly explains the effect of the gel swelling ability on the length of the initial pinned regime.

On hydrophobic SBS-paraffin gels, we have observed that the droplet contact line exhibits three different regimes of motions, and their transition is characterized by a frequency $f$ determined by the apparent contact line velocity $v$ and droplet radius $R$ as $f=v / R$. At high $f$ with respect to the cross over frequency of gel $f_{\text {cross }}$, the contact line moves continuously with a constant contact angle. As the contact line slows down and $f$ approaches to $f_{\text {cross }}$, the contact line starts the stick-slip motion: the contact line is pinned, then suddenly slips forward. At $f$ sufficiently lower than $f_{\text {cross }}$, the contact line stops stick-slip and advances continuously again. We have conjectured that the observed transitions of the contact line motions (continuous - stick-slip - continuous) are the consequence of the gel rheology affecting the dynamics of contact line. Depending on the frequency, the behaviour of the liquid contact line on gels shows both aspects of wetting on elastic solids and on viscous liquid sheets. At an intermediate frequency where the gel behaves neither solid nor liquid, the stick-slip motion appears.

Our results show that the dynamics of the contact line on gel substrates are quite different from those observed for general solid materials, especially for the appearance of the different regimes of motions. Although our experimental and theoretical approaches have 
grasped essential features, further studies are still required for the detailed understanding. For wetting on hydrophilic gels, theoretical and numerical modellings which predicts the pinned-receding transition of contact line should be realized. This requires to solve the combined equations of the water volume transport from the droplet to gel and of the balance of interfacial tensions at the contact line. Alternatively, experimental investigations of the gel surface tension are needed to provide precise parameter for these modellings [49]. For hydrophobic gels, elaborated models based on those for the wetting on elastomer should be developed. Here, we confront with more complex situations. As the gel has a visco-elastic response, two types of deformations are superposed in the gel surface ridge: reversible (elastic) and irreversible (plastic). Furthermore, the three interfacial tensions are not balanced $\left(\gamma>2 \gamma_{s}\right)$ in certain cases. These problems are remarked as one of the important future directions in wetting science.

\section{Acknowledgement}

The authors gratefully thank G. Ducouret and A. Chateauminois (ESPCIParisTech) for their supports for the rheology and surface profile measurements, Y. Shimokawa, K. Sakai (University of Tokyo) and M. Doi (Beihang University) for fruitful discussions. 
[1] E. S. Matsuo and T. Tanaka, Nature, 1990, 358, 482-485.

[2] A. Suzuki and T. Tanaka, Nature, 1990, 346, 345-347.

[3] M. Doi, J. Phys. Soc. Jpn., 2009, 78, 052001:1-19.

[4] N.A. Peppas, P. Bures, W. Leobandung and H. Ichikawa, Eur. J. Pharm. Biopharm., 2000, 50, 27-46.

[5] J. Yang, M. Yamato, C. Kohno, A. Nishimoto, H. Sekine, F. Fukai and T. Okano, Biomaterials, 2005, 26, 6415-6422.

[6] X. Hu, L. Hao, H. Wang, X. Yang, G. Zhang, G. Wang and X. Zhang, Int. J. Pol. Sci., 2011, 2011, 814163:1-9

[7] T. Baumberger, C. Caroli and D. Martina, Nature Mat., 2006, 5, 552-555.

[8] M. Morishita, M. Kobayashi, T. Yamaguchi and M. Doi, J. Phys. Condens. Matter, 2010, 22, 365104:1-6.

[9] J. P. Gong, T. Kurokawa, T. Narita, G. Kagata, Y. Osada, G. Nishimura and M. Kinjo, J. Am. Chem. Soc., 2001, 123, 5582-5583.

[10] D. Kaneko, T. Tada, T. Kurokawa, J. P. Gong and Y. Osada, Adv. Mater., 2005, 17, 535-538.

[11] D. Szabó, S. Akiyoshi, T. Matsunaga, J. P. Gong and Y. Osada, J. Chem. Phys., 2000, 113, 8253-8259.

[12] Y. Nonomura, Y. Morita, T. Hikima, E. Seino, S. Chida and H. Mayama, Langmuir, 2010, 26, $16150-16154$.

[13] Y. Nonomura, S. Chida, E. Seino and H. Mayama Langmuir, 2012, 28, 3799-3806.

[14] P. G. de Gennes, F. B. Wyart and D. Quere, Capillarity and Wetting Phenomena, Springer, 2003.

[15] D. Bonn, J. Eggers, J. Indekeu, J. Meunier and E. Rolley, Rev. Mod. Phys. 2009, 81, 739-805.

[16] M. Banaha, A. Daerr and L. Limat, Eur. Phys. J. Special Topics, 2009, 166, 185-188.

[17] D. P. Holmes, M. Roché, T. Sinha and H. A. Stone, Soft Matter, 2011, 7, 5188-5193.

[18] M. E. R. Shanahan and P. G. de Gennes, C. R. Acad. Sci. Paris, 1986, 302, Ser. II, 517.

[19] A. Carré and M. E. R. Shanahan, Langmuir, 2001, 17, 2982-2985.

[20] B. Roman and J. Bico, J. Phys.: Condens. Matter, 2010, 22, 493101:1-16.

[21] M. C. Lopes and E. Bonaccurso Soft Matter, 2012, 8, 7875-7881. 
[22] A. Aradian, E. Raphael and P.G. de gennes, Eur. Phys. J. E: Soft Matter Biol. Phys, 2000, 2, 367-376.

[23] L. Bacri and F. B. Wyart, Eur. Phys. J. E: Soft Matter Biol. Phys, 2000, 3, 87-97.

[24] K. E. Daniels, S. Mukhopadhyay, P. J. Houseworth and R. P. Behringer Phys. Rev. Lett., 2007 , 99, 124501.

[25] T. Kajiya, A. Daerr, T. Narita, L. Royon, F. Lequeux and L. Limat, Soft Matter, 2011, 7, 11425-11432.

[26] T. Kajiya, A. Daerr, T. Narita, L. Royon, F. Lequeux and L. Limat, Soft Matter, 2013, 9, 454-461.

[27] L. Limat, Eur. Phys. J. E: Soft Matter Biol. Phys, 2012 35, 134:1-13.

[28] R. P. Cámara, A. Best, H. J. Butt and E. Bonaccurso, Langmuir, 2008, 24, 10565-10568.

[29] R. P. Cámara, G. K. Auernhammer, K. Koynov, S. Lorenzoni, R. Raiteri and E. Bonaccurso, Soft Matter, 2009, 5, 3611-3617.

[30] J. Kurata, K. T. V. Grattan, H. Uchiyama and T. Tanaka, Rev. Sci. Instrum., 1990, 61, 736-739.

[31] M. Fermigier, L. Limat, J. E. Wesfreid, P. Boudinet and C. Quilliet, J. Fluid. Mech., 1992, 236, 349-383.

[32] G. Pu and S. J. Severtson, Langmuir, 2008, 24, 4685-4692.

[33] G. Pu, J. Ai and S. J. Severtson, Langmuir, 2010, 26, 12696-12702.

[34] R. D. Deegan, O. Bakajin, T. F. Dupont, G. Huber, S. R. Nagel and T. A. Witten, Phys. Rev. E: Stat., Nonlinear, Soft Matter Phys., 2000, 62, 756-765.

[35] H. Hu and R. G. Larson, J. Phys. Chem. B, 2002, 106, 1334-1344.

[36] M. Kobayashi, M. Makino, T. Okuzono and M. Doi, J. Phys. Soc. Jpn., 2010, 79, 044802:1-6.

[37] L. D. Landau and E. M. Lifshitz, Theory of Elasticity, Butterworth Heinemann, 3rd edn., 1986.

[38] S. Kundakci, Ö. B. Üzüm and E. Karadağ, Reac. Func. Polym., 2008, 68, 458-473.

[39] M. E. R. Shanahan, J. Phys. D: Appl. Phys., 1987, 20, 945-950.

[40] A. Carré, J. C. Gastel and M.E.R. Shanahan, Nature, 1996, 379, 432-434.

[41] D. Long, A. Ajdari and L. Leibler, Langmuir, 1996, 12, 1675-1680.

[42] J. C. Burton, F. M. Huisman, P. Alison, P. Rogerson and P. Taborek, Langmuir, 2010, 26, 15316-15324. 
[43] C. W. Extrand and Y. Kumagai, J. Colloid Interface Sci., 1996, 184, 191-200.

[44] E. R. Jerison, Y. Xu, L. A. Wilen and E. R. Dufresne, Phys. Rev. Lett., 2011, 106, 186103:1-4.

[45] R. W. Style and E. R. Dufresne, Soft Matter, 2012, 8, 7177-7184.

[46] A. Marchand, S. Das, J. H. Snoeijer and B. Andreotti, Phys. Rev. Lett, 2012, 108, 094301:1-4.

[47] A. J. Queimada, I. M. Marrucho and J. A. P. Coutinho, Fluid Phase Equilib., 2001, 183-184, 229-238.

[48] M. Talreja, I. Kusaka and D. L. Tomasko, Fluid Phase Equilib., 2012, 319, 67-76.

[49] Y. Yoshitake, S. Mitani, K. Sakai and K. Takagi Phys. Rev. E: Stat., Nonlinear, Soft Matter Phys., 2008, 78, 041405. 


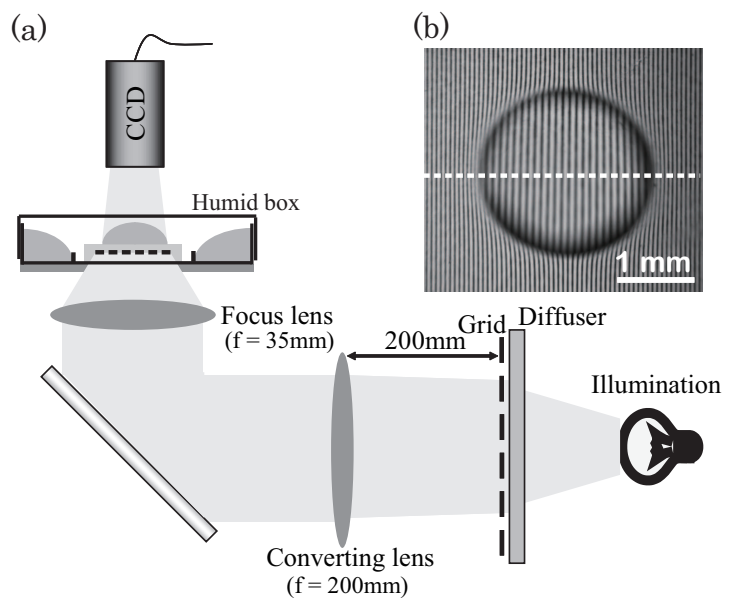

(c)

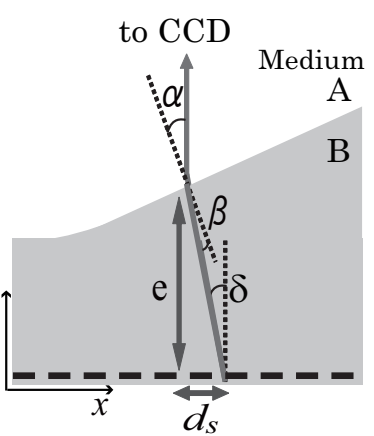

FIG. 1: (a) Schematic of grid projection technique method to obtain drop-gel profiles. (b) Example of the image of grid lines which is taken after the droplet is placed on the gel surface. (c) Geometry of the light path that passes the grid image and is detected by the CCD. 

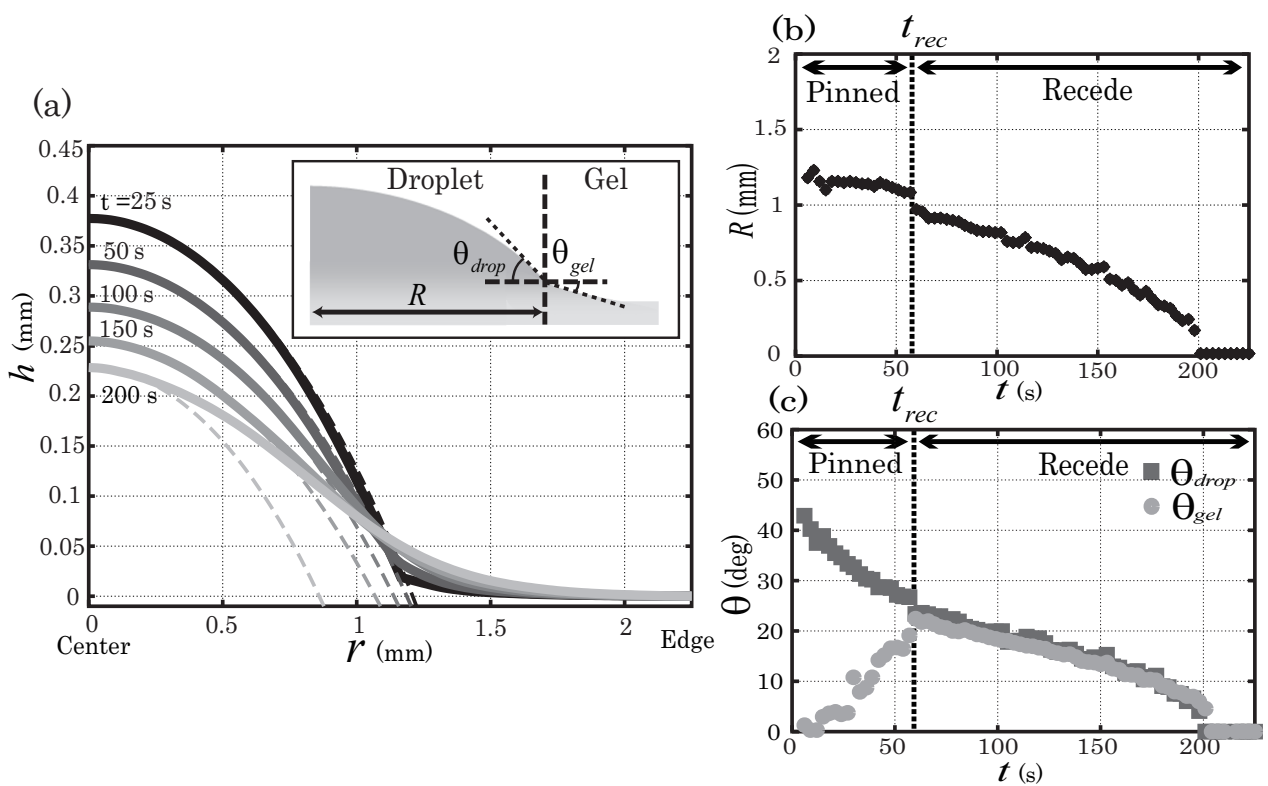

FIG. 2: Half cross sections of the profiles of the droplet and substrate $\left(C_{M B A}=5 \mathrm{~mol} \%, C_{A M P S}=\right.$ $30 \mathrm{~mol} \%$ ) at $t=25 \mathrm{~s}, 50 \mathrm{~s}, 100 \mathrm{~s}, 150 \mathrm{~s}$, and $200 \mathrm{~s}$. To detect the position of the contact line, the extrapolations of the local curvatures at the center $H_{c}$ are superposed as dashed lines. (b) Plot of the radius $R$ against the time $t$. (c) Plot of the angles of the droplet $\theta_{d r o p}$ and gel substrate $\theta_{\text {gel }}$ against $t$. The MBA concentration $C_{M B A}$ is fixed at $5 \mathrm{~mol} \%$, 
(a)

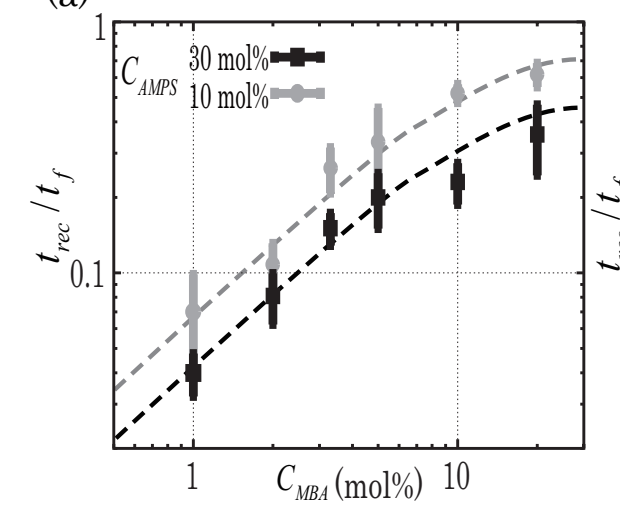

(b)

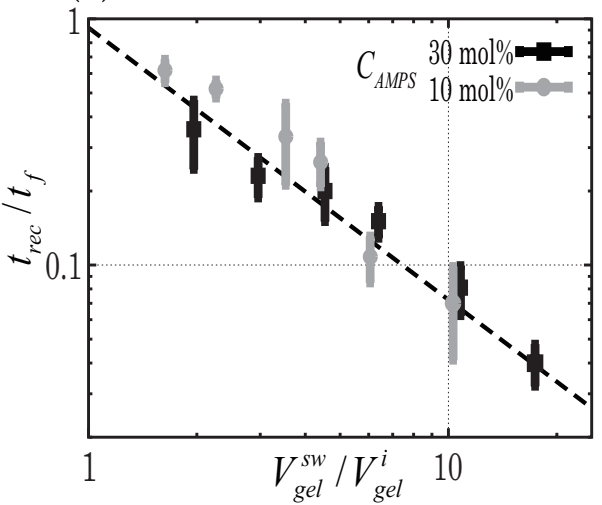

FIG. 3: (b) Plot of the relative pinning time to the total diffusion time $t_{r e c} / t_{f}$ against $C_{M B A}$. Data of two different $C_{A M P S}(10 \mathrm{~mol} \%$ and $30 \mathrm{~mol} \%)$ are plotted with different symbols. (c) Replot of $t_{r e c} / t_{f}$ against the volume swelling ratio $V_{g e l}^{s w} / V_{g e l}^{i}$. 
(a)

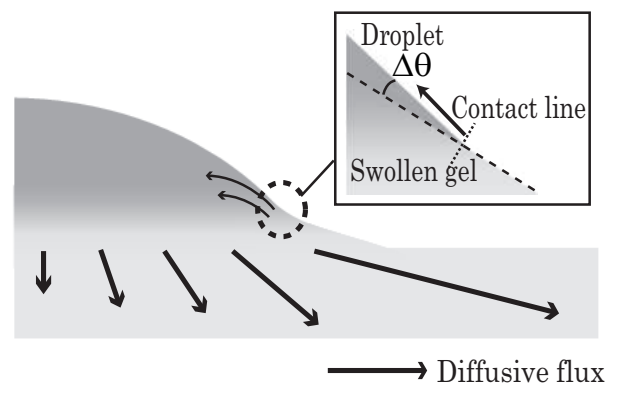

(b)

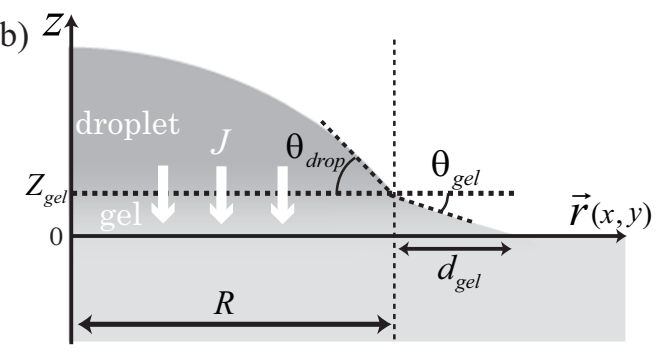

FIG. 4: (a) Mechanism of the recession of contact line. Contact line feels the locally swollen gel surface formed by diffusion of liquids. (b) Geometry and coordinates for the theoretical modelling. 
(a)

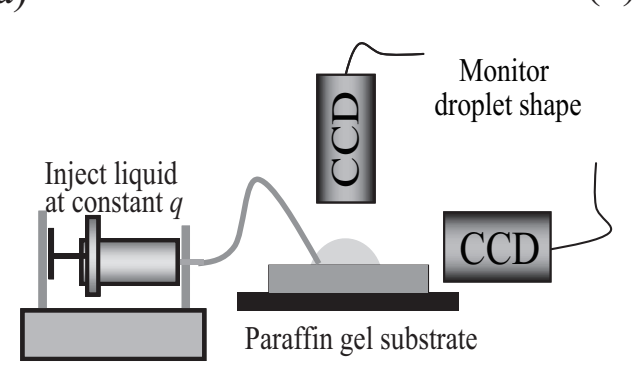

(b) From side $\begin{gathered}\text { Translate } \\ \text { at constant }\end{gathered}$

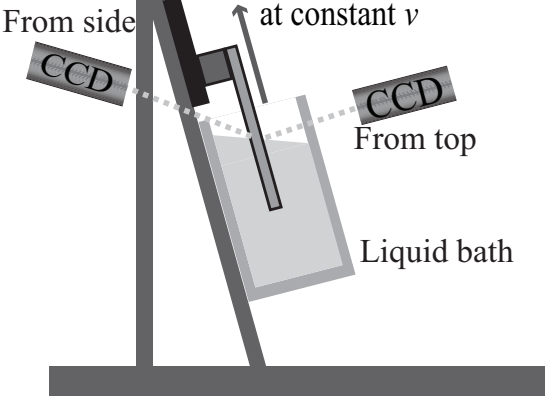

FIG. 5: Schematics of the wetting experiments on paraffin gels. (a) Inflation of sessile droplets. (b) Dip-coating. 
(a)

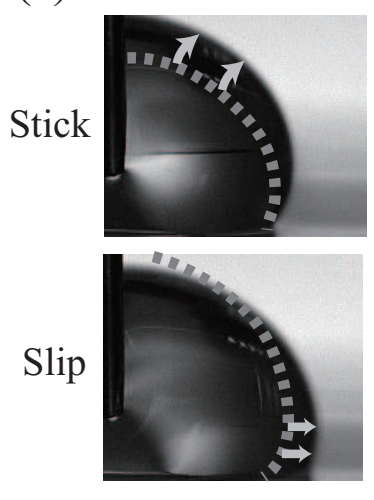

(b)

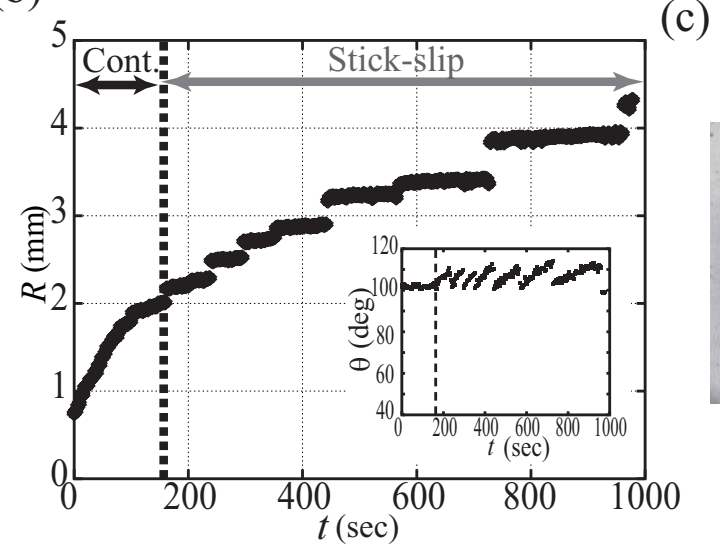

(c)

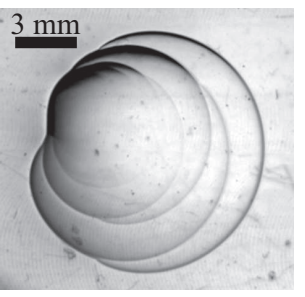

FIG. 6: (a) Stick-slip behaviour of the contact line observed in advancing droplet on SBS-paraffin gel of $c_{p o l}=10 \%$ at an inflation rate $q=20 \mu \mathrm{l} / \mathrm{min}$. (b) Plot of the radius and contact angle of the same droplet. (c) Multi-circular traces formed on the gel surface after the inflation experiment. 

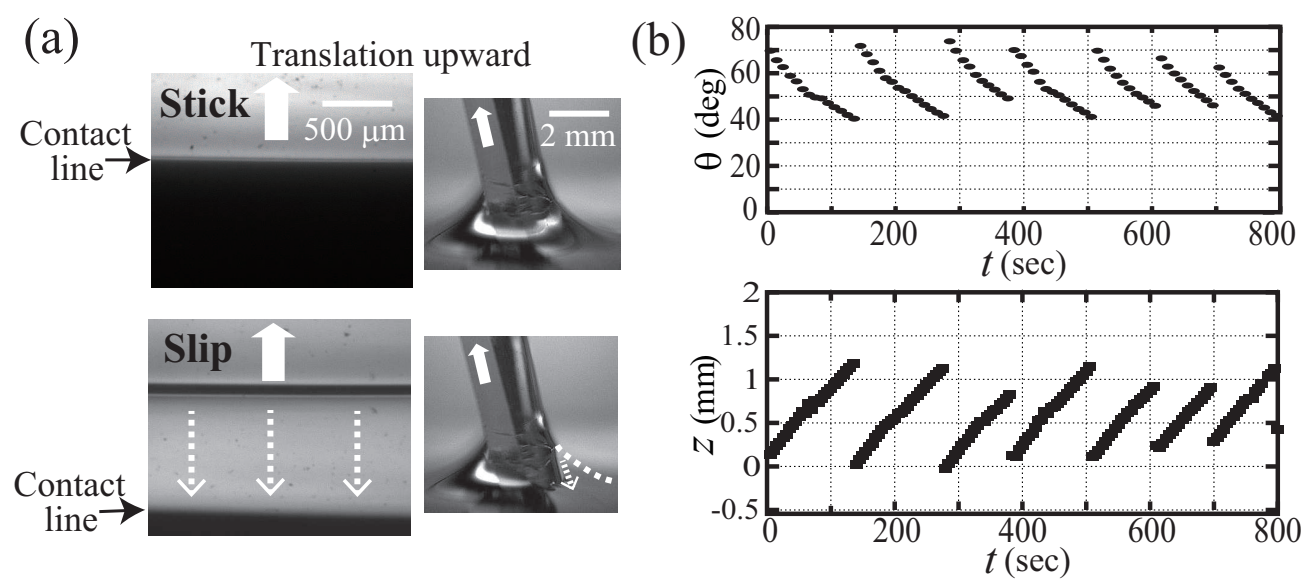

FIG. 7: (a) Stick-slip behaviour of the contact line observed in dip-coating on SBS-paraffin gel of $c_{p o l}=10 \%$ at a translation velocity $v=0.01 \mathrm{~mm} / \mathrm{s}$. The front and side views of the liquid meniscus are shown. While the substrate is withdrawn from the liquid bath at a constant velocity, the contact line of the meniscus is pinned, then suddenly slips down over a distance approximately $1 \mathrm{~mm}$. (b) Plots of the contact line position and contact angle. 


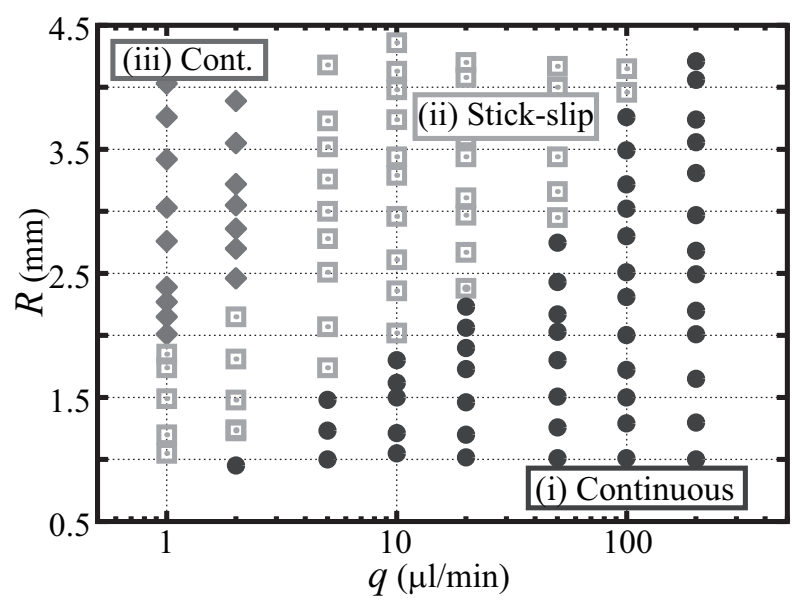

FIG. 8: Diagram of contact line behaviours as a function of $R$ and $q$. 


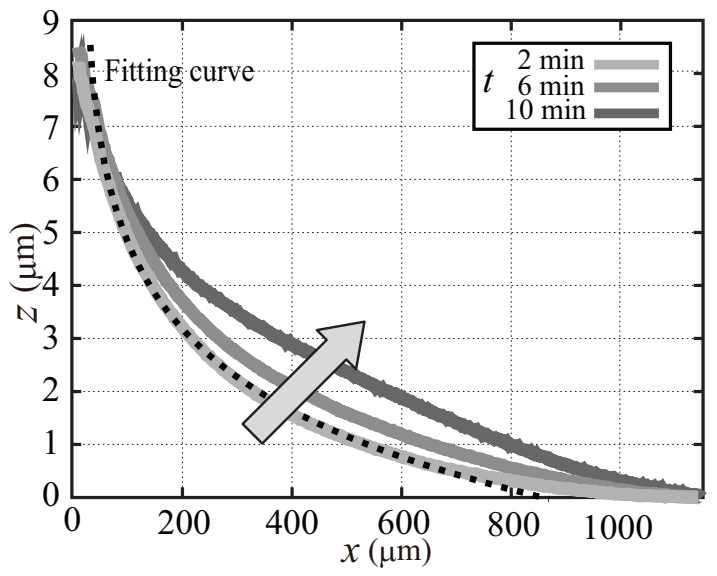

FIG. 9: Cross sections of the gel surface profiles close to the static contact line and its time evolution. A curve of the logarithmic fitting is superposed as a dashed line. 
(b)
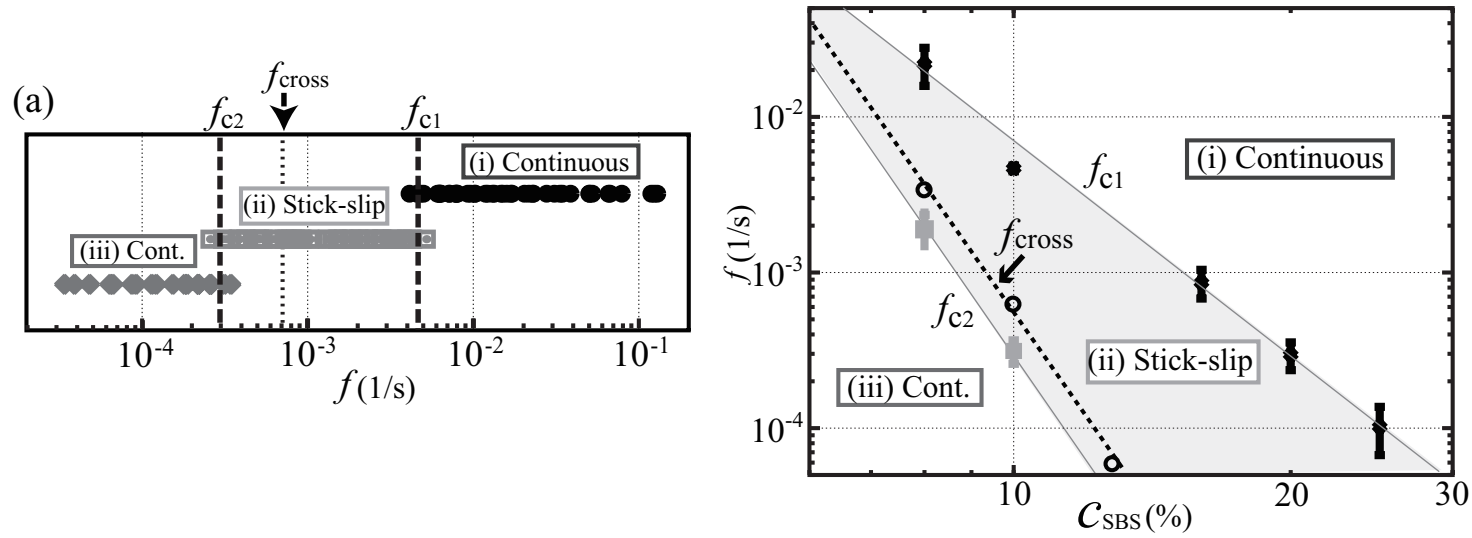

FIG. 10: (a) Replot of the data in fig. 8 against the characteristic frequency $f$. The gel crossover frequency is also shown in the figure. (b) Plot of the two critical frequencies $f_{c 1}$ and $f_{c 2}$ against SBS concentration of gel $c_{S B S}$. 
(ii) $f \approx f_{\text {cross }}$
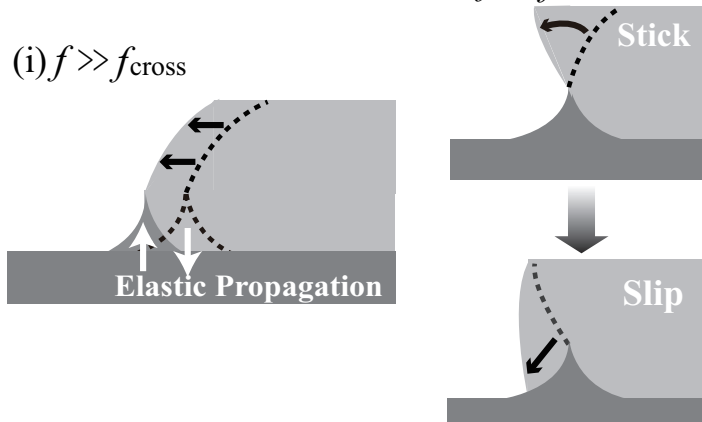

Deformation relaxes

(iii) $f \ll f_{\text {cross }}$

and pins contact line.

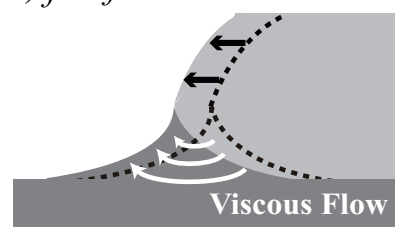

Deformation cannot

follow contact line.

FIG. 11: Behaviors of the contact line and of gel surface deformation at different regimes (i)-(iii). 

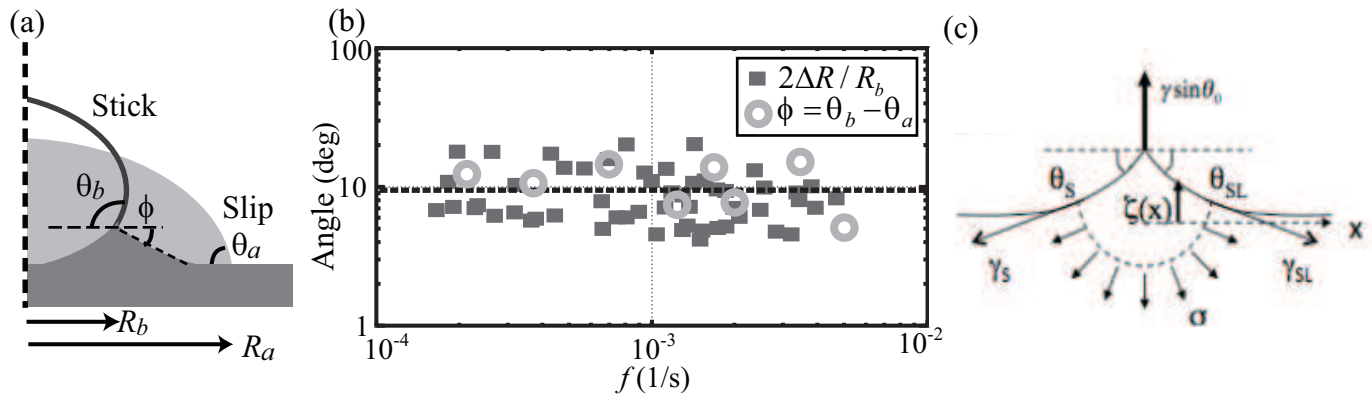

FIG. 12: (a) Parameters for the geometric considerations in the stick-slip regime. (b) Plot of the angle of the local slope of gel surface predicted from the ratio of successive droplet radii $2 \Delta R / R_{b}$ against $f$. For comparison, the value of $\phi=\theta_{b}-\theta_{a}$ directly obtained from the contact angle measurement was plotted as hollow circles. (c) Distortions induced by the normal component, that is moderated by both elastic stresses and substrate surface tensions. 